\title{
Flow dynamic study of a single-phase square NCL using recurrence plot and recurrence quantification
}

\author{
RITABRATA SAHA, KOUSHIK GHOSH, ACHINTYA MUKHOPADHYAY \\ and SWARNENDU SEN* \\ Department of Mechanical Engineering, Jadavpur University, Kolkata 700 032, India \\ *Corresponding author. E-mail: sen.swarnendu@gmail.com
}

\begin{abstract}
The cooling of a heat-generating system is one of the biggest concerns in the field of thermal engineering. Hence, the focus is on such cooling systems which do not involve any active components (passive systems) for high reliability and compact size. For this reason, in heat transfer, the natural circulation loop (NCL) is used extensively. NCL works as a cooling system by removing heat from a lower-elevation heat source and deposit the heat to a higher-elevation heat sink by the moving fluid present in it. As spontaneous dynamics of the working fluid plays a very important role in the performance of the system, it is necessary to study proper fluid flow dynamics. With increase in heater power, a change in loop fluid flow dynamics has been observed. For the comparatively low heater power, we observed steady flow, and with the increase in heater power first, we obtained the oscillatory flow, and then with the addition of more heater power, we observed flow reversal. In this study, we first investigated the instability associated with the loop fluid flow with the help of recurrence plot. Along with recurrence plot we also compute the recurrence quantification analysis. From the recurrence quantification analysis, we can predict early about the change in flow pattern from oscillatory region to flow-reversal region.
\end{abstract}

Keywords. Natural circulation loop; nonlinear dynamics; prediction of chaos; recurrence plot; recurrence quantification.

PACS Nos 05.60.-k

\section{Introduction}

Natural circulation loop (NCL) is a compact passive cooling system which has gained attention in the thermal engineering sector due to its higher reliability as well as being a safer operation. In a natural circulating system, working fluid takes heat from the heat source (at lower elevation) and becomes lighter which initiates the movement of the working fluid towards the heat sink (at higher elevation). The interplay among buoyancy, friction and gravitational forces will establish the fluid motion within the system.

As a passive heat removal system, NCL is being used in various industrial applications such as nuclear reactor core cooling, solar water heaters, gas turbine blade cooling, electrical machine rotor cooling, geothermal processes, etc., to transfer heat from one place to another and to provide better safety to the systems [1].
Due to its wide range of application, it is very important to properly characterize the flow pattern of NCL. Spontaneous dynamics of the working fluid plays a very important role in the performance of the system. Due to the low hydrodynamic head, NCLs are inherently less stable, which can cause instabilities in the flow dynamics. These instabilities may lead to failure of the system. Hence, researchers need to characterize the flow pattern and the instabilities associated with it over the past few years [2-14]. The proper identification and characterization of these instabilities are prerequisites for making an appropriate control strategy to overcome the instabilities associated with the NCL and to provide stable operation of these systems.

As single-phase NCLs have lower susceptibility to instabilities compared to two-phase NCLs, they has been used in many engineering systems. Accordingly, 
for the single-phase NCLs, a significant volume of literature exists on flow dynamics study as well as control of instability [2-14]. Numerical simulations for the fluid flow in unstable convection regime of a toroidal NCL have been observed by Ridouane et al. [2]. They found that with the increasing Rayleigh number, the flow-reversal phenomenon also increased. They studied streamlines, isotherms and local heat flux distributions for each of the flow regime. They also observed the time series data of the heat flux distribution along the walls during flow reversals and found that heat flux distribution showed a very complex distribution. With the help of an analytical model, stability analysis by stability mapping of a single-phase thermosyphon loop has been done by Maiani et al. [3]. To investigate the flow transitions and instabilities numerical simulations have been performed by Chen et al. [5], for the NCL which is filled with supercritical $\mathrm{CO}_{2}$. They used temperaturesensitive physical properties to calculate the stabilities of the system. From their study, it was found that due to the supercritical $\mathrm{CO}_{2}$ as loop fluid, with the increase in source temperature, fluid flow dynamics changed from unstable flow-reversal nature to stable one-directional flow.

To observe the fluid flow dynamics and to find out the stable regime, both experimental and numerical studies have been done by Vijayan et al. [6]. They found experimentally that the fluid flow dynamics could be divided into three categories, but numerically, only two different categories were found. From their study, it was also observed that fluid flow dynamics behaved like conditionally stable near the stability threshold. Stability characteristics for the rectangular NCL fluid flow has been mathematically simulated by Nayak et al. [8]. They identified the stable, unstable and neutrally stable points by examining the amplitude of flow and temperature oscillations with time with the help of Nyquist stability criterion. Stability behaviour was also analysed by the linear stability theory and comparison of these two results were done by the stability mapping.

Cammarata et al. [9] observed fluid flow dynamics for NCL using nonlinear analysis tools for the experimental time series data. They used Phase Space Reconstruction, Singular Value Decomposition and Average Mutual Information techniques as the nonlinear analysis tools to study the dynamics behaviour. Vijayan et al. [10] performed different experiments along with the numerical investigation using the computer code ATHLET to observe how the instability grows with the increase in loop diameter. They also studied how the fluid flow dynamics changes with the increase in power level. A computer code SUCLIN was developed by Sharma et al. [11] for the steady state and linear stability analysis of supercritical water natural circulation loop (SCWNCL). Also, the effect of various parameters like loop diameter, loop height, heater inlet temperature, pressure and local loss coefficients on SCWNCL were investigated.

Nayak et al. [12] experimentally observed that the flow instability associated with the NCL was significantly reduced by the use of small concentration of $\mathrm{Al}_{2} \mathrm{O}_{3}$ nano-fluid suspended into the water. Also, it was observed that in single-phase mass flow rate has been increased significantly due to the presence of small concentration of $\mathrm{Al}_{2} \mathrm{O}_{3}$ nano-fluid. But, with the increasing concentration of $\mathrm{Al}_{2} \mathrm{O}_{3}$, flow instability increased. Mathematical modelling to simulate the single phase NCL using RELAP5 system code and with a developed computer program was done by the Mousavian et al. [13]. For the stability analysis, they used stability mapping with the help of Nyquist stability criterion. Loop fluid flow dynamics study for a rectangular NCL by two-dimensional numerical analysis was performed by Desrayaud et al. [14]. They observed the effect of Rayleigh number on the loop fluid flow dynamics. Loop fluid flow dynamics study for an annular thermosyphon was investigated experimentally and numerically by Desrayaud et al. [15].

Although a large volume of work is available on instability associated with NCL, very few works have been reported that systematically characterize the fluid flow dynamics using quantitative tools of nonlinear dynamics. Zhang et al. [16] characterized the time series data obtained from natural circulation using quantitative tools of nonlinear dynamics but their study was limited to oscillatory region and did not provide any information about the flow reversal region. Nishikawa et al. [4] characterized the time series data obtained from rectangular natural convection loop using recurrence plot, but the detailed recurrence quantification analysis is not present there.

In the present study, we tried to identify the flow patterns obtained from the numerical simulations. The simulations were carried out with a SIMULINK model developed in our previous works [17, 18]. With the increase in heater power, flow pattern of the working fluid changed from steady state region to flow-reversal region through oscillatory region. To characterize the loop fluid flow dynamics, we used recurrence plot as nonlinear dynamic analysis tool. This characterization is very important to distinguish between possibly chaotic systems, which are deterministic in nature, from those dominated by noise, which is stochastic in nature. As the control strategy of deterministic system and stochastic system are different, this characterization will help us to make appropriate control strategy. Along with recurrence plot we also carry out the recurrence quantification 
analysis. From the recurrence quantification analysis, we can predict the change in the flow pattern from oscillatory region to flow reversal region. This prediction will help us to detect when control strategy needs to be fired.

\section{Numerical modelling}

Numerical model and the geometry (figure 1), used here, was described in detail in our previous paper [18]. Conservation of mass, momentum and energy for the loop fluid are shown in eq. (1) to eq. (3) respectively. The energy equations for coolant and tube wall are shown in eq. (4) and eq. (5) respectively. This equations are similar to our earlier works $[17,18]$, therefore we are avoiding repeated explanations of the equations for the sake of brevity.

$$
\begin{aligned}
& \frac{\partial \rho_{f}}{\partial t}+\frac{\partial}{\partial z}\left(\left(\rho v_{f}\right)\right)=0 \\
& \frac{d \rho v_{f}}{d t} L_{\text {loop }}=-\frac{f_{\text {in }}(\rho)_{f}^{2}}{2 \rho_{f} d_{\text {in }}} L_{\text {loop }}+\oint \rho_{0} g \beta_{a v}\left(T_{f}-T_{\text {ref }}\right) d z
\end{aligned}
$$

$$
\begin{gathered}
\frac{\partial}{\partial t}\left(\rho_{f} C_{p} T_{f}\right)+\frac{\partial}{\partial z}\left((\rho v)_{f} C_{p} T_{f}\right) \\
=\frac{\partial}{\partial z}\left(k_{f} \frac{\partial T_{f}}{\partial z}-\frac{4 h_{i}\left(T_{f}-T_{w}\right)}{d_{i n}}\right) \\
\frac{\partial}{\partial t}\left(\rho_{e x} C_{p e x} T_{e x}\right)+\frac{\partial}{\partial z}\left((\rho v)_{e x} C_{p e x} T_{e x}\right) \\
=\frac{\partial}{\partial z}\left(k_{e x} \frac{\partial T_{e x}}{\partial z}\right)+\frac{h_{e x} A_{0}\left(T_{w}-T_{e x}\right)}{V_{e x}} .
\end{gathered}
$$

The heat conduction equation for tube wall is

$$
\begin{gathered}
\frac{\partial}{\partial t}\left(\rho_{w} C_{p w} T_{w}\right)=\frac{\partial}{\partial z}\left(k_{w} \frac{\partial T w}{\partial z}\right)+\frac{h_{i} A_{i}\left(T_{f}-T_{w}\right)}{V_{w}} \\
-\frac{h_{0} A_{0}\left(T_{w}-T_{a}\right)}{V_{w}}+\frac{\dot{Q}}{V_{w}} .
\end{gathered}
$$

To solve these governing equations, several closure relations were implemented, which incorporates the friction factor $(f)$, heat transfer co-efficient between wall and loop fluid, between wall and coolant in the heat exchanger and between wall and outside air. For evaluation of Friction Factor $(f)$, in the SIMULINK model, we used:

$f=64 / R e_{d}$ for $R e_{d}<2300$

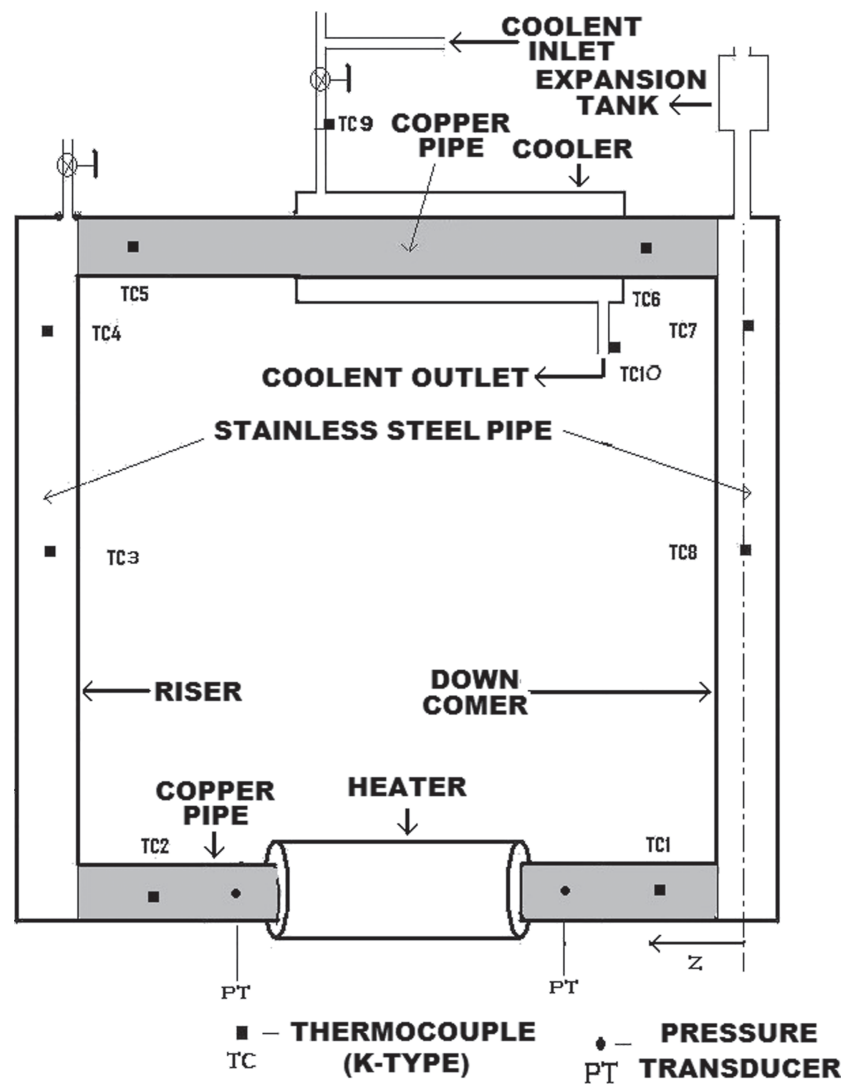

Figure 1. Schematic diagram of square natural circulation loop $[17,18]$.

$$
\begin{gathered}
f=3.03 \times 10^{-12} R e_{d}^{3}-3.67 \times 10^{-8} R e_{d}^{2} \\
+1.46 \times 10^{-4} R e_{d}-0.151 \\
\quad \text { for } 2300<R e_{d}<4500
\end{gathered}
$$

$f=\left(0.79 \ln R e_{d}-1.64\right)^{-2} \quad$ for $R e_{d}>4500$,

where $R e_{d}$ is the Reynolds number given by

$\operatorname{Re}_{d}=\frac{G_{f} d_{i n}}{\mu_{f}}$

The correlations used for determining fluid to wall heat transfer are shown below.

For the laminar flow,

$$
N u_{d}=1.86 \operatorname{Re}_{d}^{\frac{1}{3}} p r^{\frac{1}{3}}\left(\frac{d_{\text {in }}}{L_{\text {loop }}}\right)^{\frac{1}{3}}\left(\frac{\mu}{\mu_{\text {bulk }}}\right)^{0.14} \text { for } G z>10
$$

$N u_{d}=3.66$ for $G z \leq 10$ 
Here Graetz Number $(\mathrm{Gz})$ is defined as

$G z=\frac{\pi d_{\text {in }}}{4 L_{\text {loop }}} \operatorname{Re} e_{d} \operatorname{Pr}$.

We are using Petukhov correlation with Gnienlinski modification for determining Nusselt number for the transition and turbulent flow, with corresponding friction factors as given by eqs (7) and (8).

$N u_{d}=\frac{\left(\frac{f}{8}\right)\left(R e_{d}-1000\right) \operatorname{Pr}}{1+12.7(f / 8)^{1 / 2}\left(\operatorname{Pr}^{2 / 3}-1\right)}$.

For estimating the heat transfer from tube wall to ambient for the riser and down-comer sections, the following correlations of vertical cylinders we used.

$R a=\frac{g \beta L_{\text {loop }}^{3}\left(T_{w}-T_{\alpha}\right)}{v \alpha}$,

For vertical cylinders:

$N u_{l, c y l}=C F * N u_{1}$,

where

$N u_{1}=\frac{2.8}{\ln \left(1+\frac{2.8}{C_{1} R a^{1 / 4}}\right)}$,

$R a=G r . P r=\frac{g \beta L_{\text {loop }}^{3}\left(T_{w}-T_{\alpha}\right)}{v^{2}} \frac{v}{\alpha}$,

where

$C F=\frac{1.8 \varphi}{\ln (1+1.8 \varphi)} \quad$ and $\quad \varphi=\frac{L / d_{0}}{C_{1} R a^{1 / 4}}$

The heat loss for the bare tube walls to the ambient is estimated by the following correlation,

$$
N u_{1}=\left(0.60+\frac{0.387 R a^{\frac{1}{6}}}{\left[1+\left(\frac{0.559}{p r}\right)^{\frac{9}{16}}\right]^{\frac{8}{27}}}\right)^{2} 10^{-5}<R a<10^{12} .
$$

\section{Validation and solution procedure}

The numerical model we are using in the present work was validated with the results of Vijayan et al. [7]. The comparison is available in the figure 9 of our earlier work [18] and not repeated here for brevity. Also the grid and time independence study is available in our previous work [18] and for the compactness is not presented here again.

Recurrence plot is a dynamic systems analysis tool, which has been used in various fields to properly characterize the time series data [4,19-25]. For a nonlinear system like NCL, phase plot and Fast Fourier Transform (FFT) study are not sufficient for the proper characterization of the flow dynamics. Therefore, to characterize the flow dynamics, we used Recurrence plot as the nonlinear dynamic analysis tool. This characterization is very important to distinguish between possibly chaotic systems, which are deterministic in nature from those dominated by noise, which is stochastic in nature. As the control strategy of deterministic system and stochastic system are different, this characterization will help us to make appropriate control strategy. To find out recurrence plot, first we need to convert time series data into phase space data. In the reconstructed phase space, proximity of two state vectors, one being $x_{i}=$ $\left[y\left(t_{i}\right), y\left(t_{i}+\tau\right), \ldots, y\left(t_{i}+\left(d_{E}-1\right) \tau\right)\right]$ and the other being $x_{j}=\left[y\left(t_{j}\right), y\left(t_{j}+\tau\right), \ldots, y\left(t_{j}+\left(d_{E}-1\right) \tau\right)\right]$, can be expressed in terms of recurrence matrix [23,24]. Here, $y\left(t_{i}\right)$ represents the instantaneous value of a scalar variable and $\tau$ and $d_{E}$ represents the delay time and embedding dimension respectively. Recurrence matrix can be evaluated in various ways. The most significant way is identifying the vectors about a given vector e within a fixed distance (a hyper sphere of radius $\varepsilon$ ) of the vector. The recurrence matrix is a binary matrix represented as [23]:

$R i j=H\left(\varepsilon-\left\|x_{i}-x_{J}\right\|\right)$.

Here $H$ denotes Heaviside function. Graphically representing $R_{i j}=0$ or 1 as white and black dot's respectively, and recurrence plots can be generated. The principal diagonal consists of black dots only as $R_{i i}=1$ always and is referred to as the Line of Identity (LOI). The appearance of the recurrence plot gives important qualitative information about the dynamic characteristics of the system. Equally spaced lines parallel to the LOI represent a periodic system. The distance between the lines represents the time period of the system. Short diagonal line segments, parallel to the LOI indicate noisy periodic behaviour or quasi-periodic behaviour, with the gaps created because of noise. The vertical lines represent intermittent steady behaviour. A 
box-like structure in the recurrence plots represents the chaotic systems and recurrence plots for the stochastic time series data is represented by white and black dots distributed randomly. Beyond this graphical information about the recurrence plots, more detailed information can be obtained from the recurrence quantification analysis as mentioned below.

\subsection{Recurrence rate}

Recurrence rate denotes the fraction of recurrence or neighbouring points in the phase space and is defined as

$R R=\frac{1}{M^{2}} \sum_{i, j=1}^{M} R_{i j}$.

Here $M$ is the length of the time series data and $R_{i j}$ is the recurrence matrix.

\subsection{Determinism}

Determinism denotes the fraction of the recurring points that form diagonal structures parallel to the LOI and is denoted by

$D E T=\frac{\sum_{l=l \min }^{M} l P(l)}{\sum_{i, j=1}^{M} R_{i j}}$

$P(l)$ denotes the number of diagonal lines of length $l$ (in terms of the number of successive points along a diagonal for which $\left.R_{i j}=1\right)$. Thus, $l P(l)$ denotes the number of recurrence points in diagonal structures of length $l . l_{\min }$, is the minimum length of a segment to be considered as a diagonal structure.

The average length of a diagonal line gives an estimate of the average time for which two segments of a trajectory stay close to each other is given by

$L=\frac{\sum_{l=l_{\min }}^{M} l P(l)}{\sum_{l=l_{\min }}^{M} P(l)}$.

The average length of the diagonal line is a measure of the divergence of trajectories and is, thus, inversely proportional to Lyapunov exponent.

\subsection{Laminarity}

Laminarity is a measure of the fraction of recurrence points forming vertical structures and is given by

$L A M=\frac{\sum_{v=v_{\min }}^{N} v P(v)}{\sum_{v=1}^{N} v P(v)}$.
Vertical structures in recurrence plots indicate proximity of states (points in phase space) at successive time instants. Here $P(v)$ denotes the number of vertical lines of length $v \cdot v_{\min }$ is the minimum length of a segment to be considered as a vertical line.

\section{Results and discussion}

From the numerically obtained time series data, system dynamics can be segregated into three categories. For heater power, less than $625 \mathrm{~W}$, loop fluid flow dynamics gives steady state behaviour. With the increase in heater power upto $742 \mathrm{~W}$, an oscillatory flow is observed. For further increase in heater power, fluid flow dynamics shows flow-reversal phenomenon. For identifying the fluid flow dynamics quantitatively, we analysed the results after the initial transient part was overcome.

Fluid flow in a NCL is governed by the temperature difference $(\Delta T)$ between its two vertical limbs, the riser (having higher temperature) and the down-comer (having lower temperature). The temperature difference between these two limbs causes a density difference, and this in turn causes fluid flow due to the balance of buoyancy, friction and gravitational forces.

For heater power of $500 \mathrm{~W}$, the variation of temperature-difference between midpoints of two vertical limbs with time is shown in figure 2. From the figure, it can be observed that the temperature difference shows the steady state behaviour.

For heater power of $660 \mathrm{~W}$ and $720 \mathrm{~W}$ the variation of temperature difference with time is shown in figures 3 and 4 respectively. From the figures, it can be observed that temperature difference shows oscillatory flows which are probably periodic in nature. Also

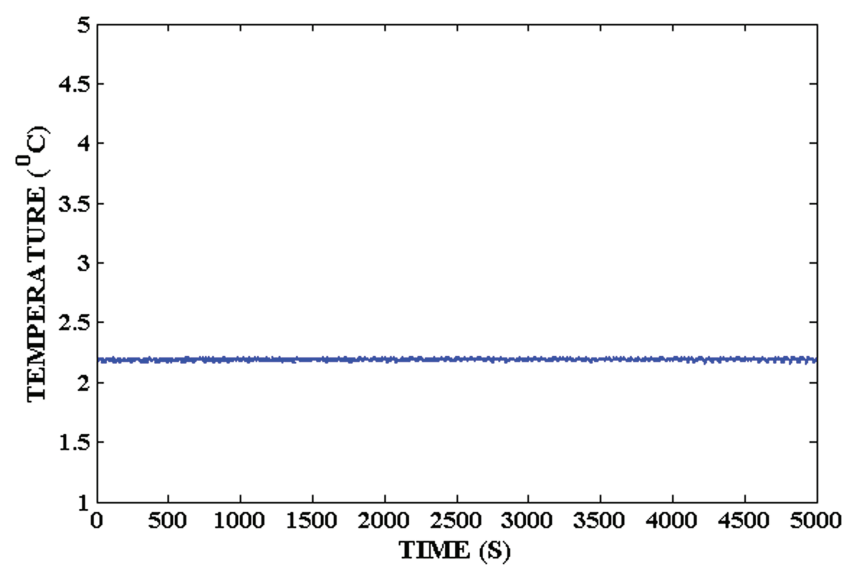

Figure 2. Variation of temperature difference with time at $500 \mathrm{~W}$. 


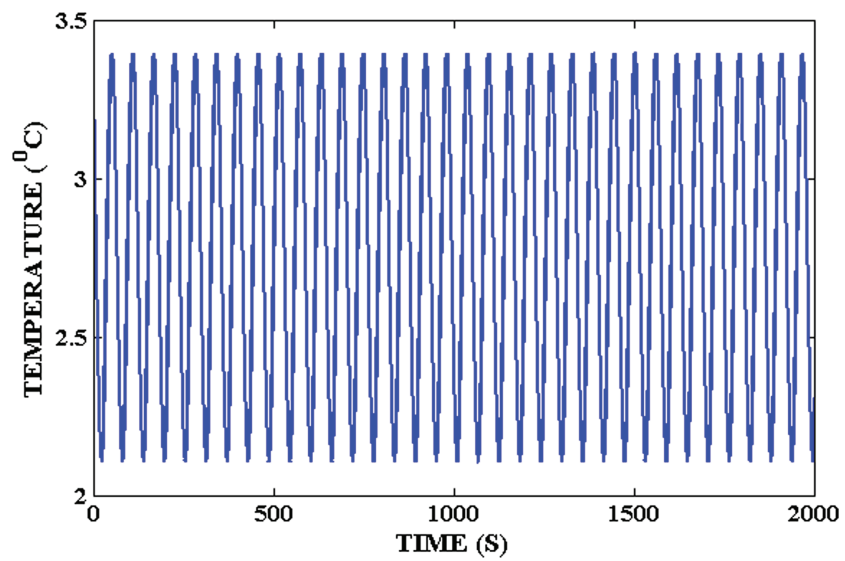

Figure 3. Variation of temperature difference with time at $660 \mathrm{~W}$.

from the comparative study of figures 3 and 4, it can be observed that the oscillation amplitude increases with the increase in heater power. For heater power of $660 \mathrm{~W}$, the oscillation amplitude of temperature difference (difference between maximum and minimum temperature difference) is about $1.3^{\circ} \mathrm{C}$ while for heater power $720 \mathrm{~W}$, the oscillation amplitude increases to about $2.7^{\circ} \mathrm{C}$.

For heater power of $745 \mathrm{~W}$ and $780 \mathrm{~W}$ the variation of temperature difference is shown in figures 5 and 6 . From the figures, it can be observed that the temperature difference between midpoints of two vertical limbs shows a flow-reversal phenomenon which is probably chaotic in nature. Also, it can be observed that the frequency of flow-reversal occurrence increases with the increasing heater power.

To acquire recurrence plot, first we need to evaluate the time delay and embedding dimension. The optimal time delay was evaluating by autocorrelation function

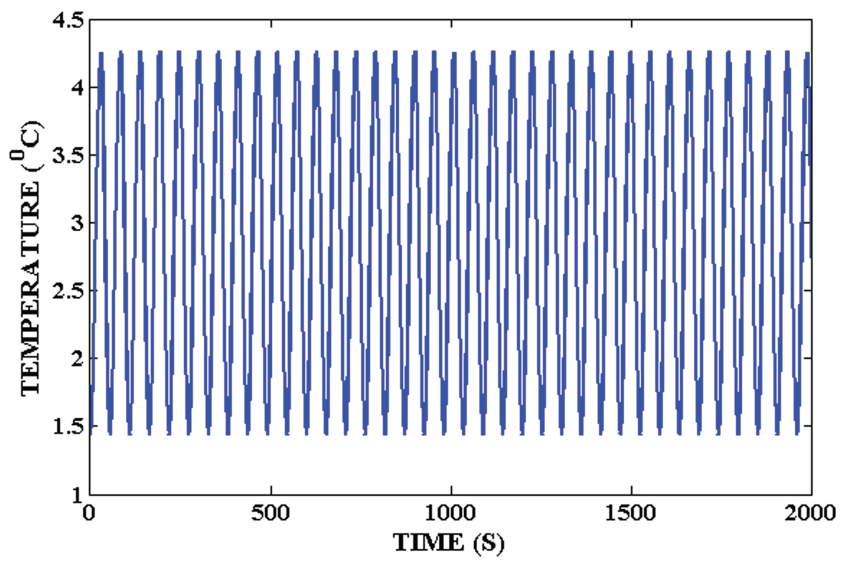

Figure 4. Variation of temperature difference with time at $720 \mathrm{~W}$.

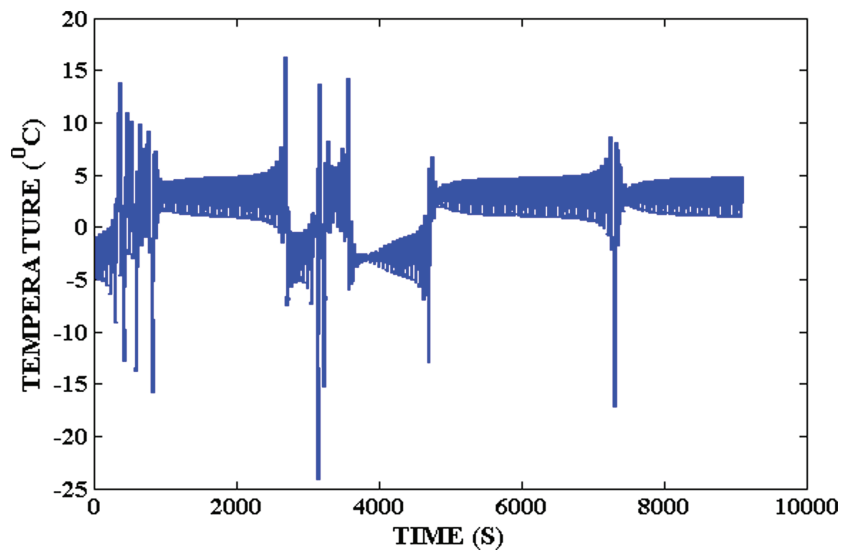

Figure 5. Variation of temperature difference with time at $745 \mathrm{~W}$.

and the embedding dimension was evaluating from correlation dimension [26]. We used temperature difference time series data to construct recurrence plot and performing recurrence quantification analysis. To obtain recurrence matrix, we used threshold $(\varepsilon)$ as 0.05 times of the maximum distance between $x_{i}$ and $x_{j}$. We found that this threshold value is sufficiently small so that we could get proper results. Recurrence plots for heater power $660 \mathrm{~W}$ and $720 \mathrm{~W}$ are shown in figures 7 and 8 , respectively. From the figures, it can be observed that the recurrence plot shows equally spaced diagonal line structure parallel to the LOI, which signify the periodic nature. Therefore, we can say that the oscillations in the time series data in the oscillatory region are purely periodic.

Recurrence plots for heater power $745 \mathrm{~W}$ and $780 \mathrm{~W}$ are shown in figures 9 and 10, respectively. From the figures, it can be observed that the recurrence plot shows box like structure, which signify the chaotic nature.

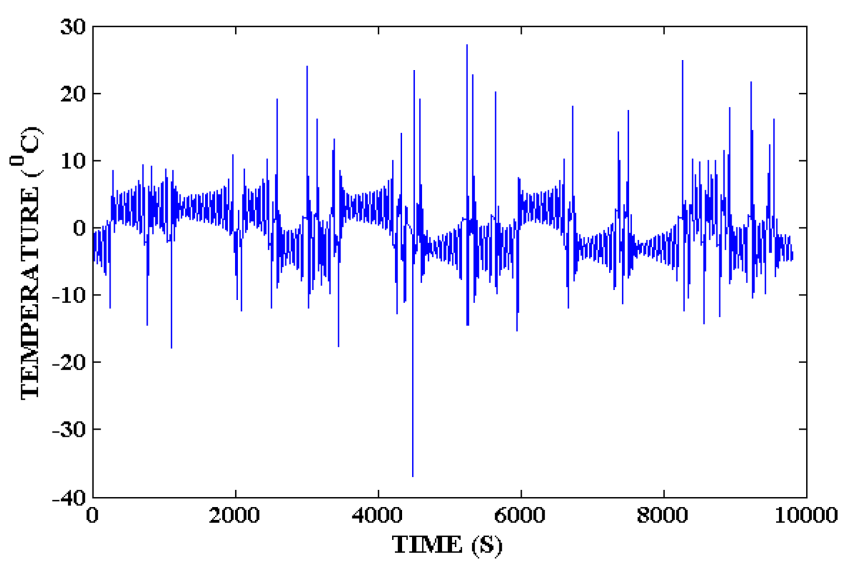

Figure 6. Variation of temperature difference with time at $780 \mathrm{~W}$. 


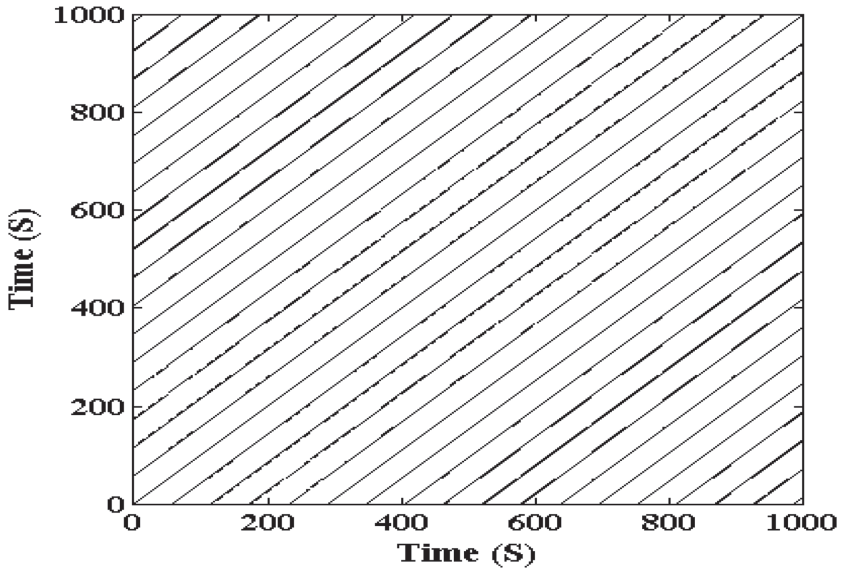

Figure 7. Recurrence plot at $660 \mathrm{~W}$.

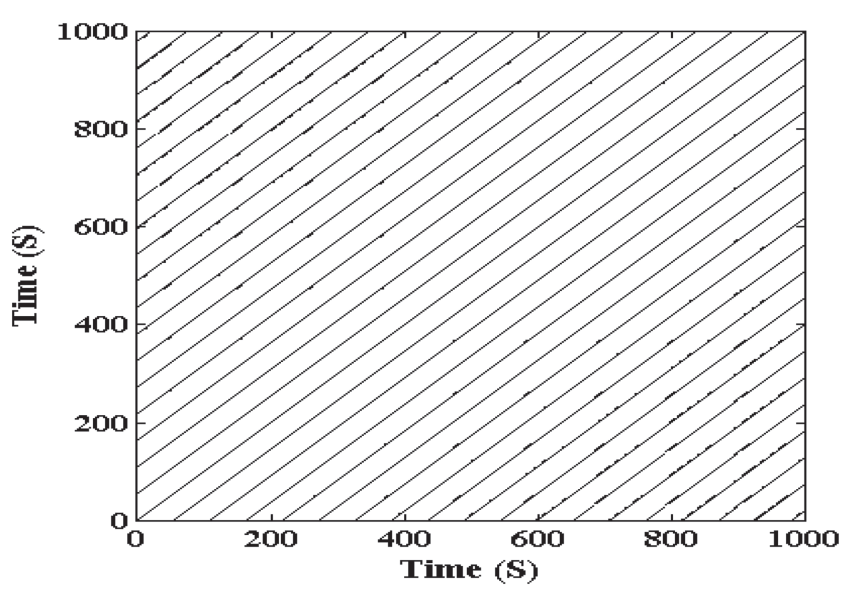

Figure 8. Recurrence plot at $720 \mathrm{~W}$.

Therefore, we can say that the fluid flow dynamics of the flow reversal region are chaotic. To determine the proper control strategy, confirmation of chaotic behaviour is very important.

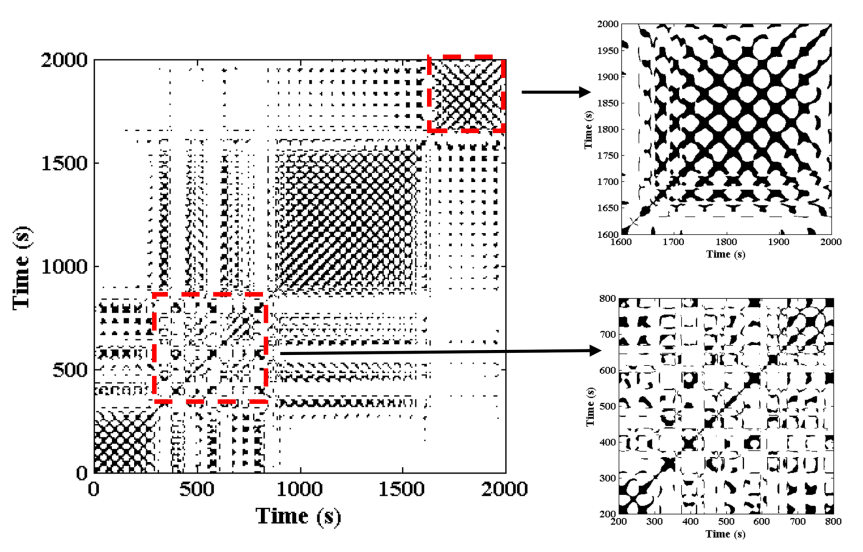

Figure 9. Recurrence plot at $745 \mathrm{~W}$.

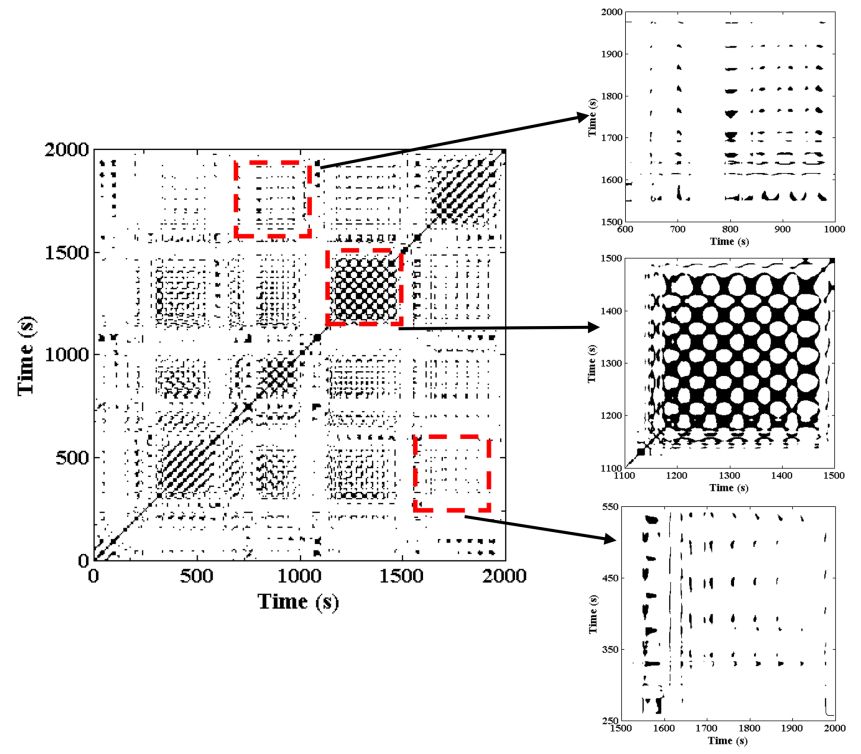

Figure 10. Recurrence plot at $780 \mathrm{~W}$.

For practical applications like power plants which use NCL, flow reversal is an undesirable phenomenon. Therefore, it is required to avoid flow-reversal condition. Flow reversals occur at irregular intervals, and with the increase in heater power, frequency of flow-reversal occurrence increases. Therefore, to suppress these flow dynamics we need to use active feedback control strategies. The recurrence quantification analysis, such as recurrence rate as shown in figure 11 , determinism as shown in figure 12 and laminarity as shown in figure 13, can be used to predict the change in the flow pattern from oscillatory region to flow-reversal region.

From figure 11 we can observe that recurrence rate stays a very low and constant value for the periodic regime. This jumps to a higher value at the onset of flow reversal with an increase in heater power. In this chaotic

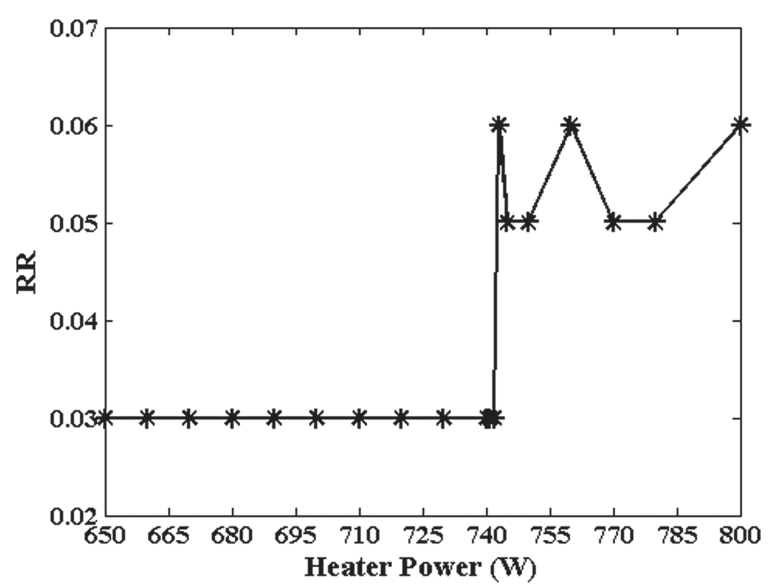

Figure 11. Variation of recurrence rate with heater power. 


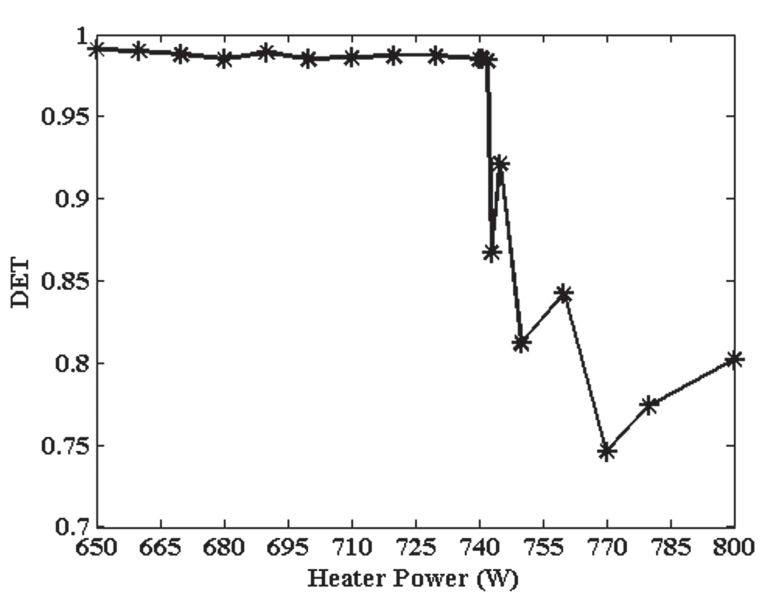

Figure 12. Variation of determinism with heater power.

regime, it shows an unstable nature. From figure 12 we can observe that determinism stays at a roughly steady value (close to 1 ) for the periodic regime. But for the chaotic regime, it starts decreasing and shows an unstable low value. From figure 13 we can observe that laminarity stays at a constant value (zero) for the periodic regime. But, at the end of this regime, when we move to the chaotic region, laminarity starts increasing from zero and became unstable and poses very high value (close to 1 ).

Among the three properties, coming out of recurrence quantification, laminarity shows a nature which can be used for early detection of flow reversal. As the laminarity value starts changing from steady value (zero), it can be predicted that the situation is close to flow reversal. Accordingly, the control strategies can be invoked to avoid flow reversal. However, the other two properties do not show such a trend in their plot to be used for the prediction of flow reversal.

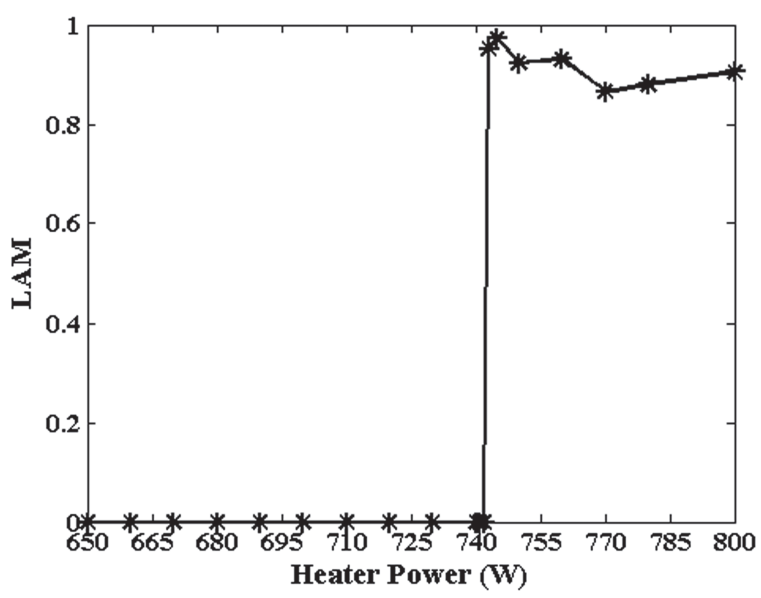

Figure 13. Variation of laminarity with heater power.

\section{Conclusion}

From the numerical study, it is observed that fluid flow dynamics can be divided into three categories. We get stable steady state dynamics of heater power less than $625 \mathrm{~W}$, and for heater power $625 \mathrm{~W}$ to $742 \mathrm{~W}$, we get a stable oscillatory flow, and above this heater power, we get flow-reversal characteristics.

From the recurrence plot analysis, it is confirmed that the fluid flow dynamics in the oscillatory region are purely periodic, and accordingly recurrence plots show equally spaced diagonal line structure. From the recurrence plot analysis, it can be concluded that the fluid flow dynamics in the flow-reversal region are chaotic, and accordingly recurrence plots show box-like structure.

From the recurrence quantification study, for the periodic regime, we obtained a steady value, but when closer to the chaotic regime, the steady, stable value starts deviating and in the chaotic regime we get fluctuating values. With the help of laminarity, we can predict the starting of chaos early in order to take control action.

\section{Acknowledgements}

RS is grateful to UGC for providing the fellowship under UPE - Phase-II Program. The authors gratefully acknowledge the helpful discussions with Dr. Sirshendu Mondal, IIT Madras, and Uddalok Sen, University of Illinois, regarding dynamic characterization tools.

\section{Appendix: Nomenclature}

A Area of crosssection $\left(\mathrm{m}^{2}\right)$

$\mathrm{A}_{\mathrm{in}} \quad$ Internal crosssection area of loop $\left(\mathrm{m}^{2}\right)$

$\mathrm{A}_{0} \quad$ External crosssection area of loop $\left(\mathrm{m}^{2}\right)$

$\mathrm{C}_{\mathrm{p}} \quad$ Specific heat of fluid at constant pressure $(\mathrm{J} / \mathrm{kg} \cdot \mathrm{K})$

$\mathrm{C}_{\mathrm{pw}} \quad$ Specific heat of wall at constant pressure $(\mathrm{J} / \mathrm{kg} \cdot \mathrm{K})$

$\mathrm{C}_{\text {pex }} \quad$ Specific heat of coolant at constant pressure $(\mathrm{J} / \mathrm{kg} \cdot \mathrm{K})$

$\mathrm{d}_{\text {in }} \quad$ Internal loop diameter $(\mathrm{m})$

$\mathrm{Dz} \quad$ Change in length (m)

F Friction factor

Gr Grashof number

$\mathrm{Gr}_{\mathrm{m}} \quad$ Modified Grashof number $\left(\mathrm{D}^{3} \rho_{\mathrm{f}}^{2} \beta_{\mathrm{av}} \mathrm{gL} \mathrm{L}_{\mathrm{H}} \dot{Q}\right) /\left(\mathrm{A} \mu_{\mathrm{f}}^{3} \mathrm{C}_{\mathrm{p}}\right)$

$\mathrm{Gz} \quad$ Graetz number

$\mathrm{Nu} \quad$ Nusselt number 


$\begin{array}{ll}\mathrm{Pr} & \text { Prandtl number } \\ \mathrm{T} & \text { Time }(\mathrm{Sec}) \\ \mathrm{T}_{\mathrm{f}} & \text { Temperature of fluid }\left({ }^{\circ} \mathrm{C}\right) \\ \mathrm{T}_{\mathrm{w}} & \text { Loop wall temperature }\left({ }^{\circ} \mathrm{C}\right) \\ \mathrm{T}_{\mathrm{ex}} & \text { Coolant temperature }\left({ }^{\circ} \mathrm{C}\right) \\ \mathrm{T}_{\mathrm{ref}} & \text { Reference temperature }\left({ }^{\circ} \mathrm{C}\right) \\ \mathrm{T}_{\mathrm{a}} & \text { Ambient temperature }\left({ }^{\circ} \mathrm{C}\right) \\ \mathrm{Ra} & \text { Rayleigh number } \\ \mathrm{h}_{\mathrm{i}} & \text { Heat transfer coefficient between fluid } \\ & \text { \& wall }\left(\mathrm{W} / \mathrm{m}^{2} \cdot \mathrm{K}\right) \\ \mathrm{h}_{\mathrm{o}} & \text { Heat transfer coefficient between wall } \\ & \text { \& ambient }\left(\mathrm{W} / \mathrm{m}^{2} \cdot \mathrm{K}\right) \\ \mathrm{h}_{\mathrm{ex}} & \text { Heat transfer coefficient between wall } \\ & \text { \& coolant }\left(\mathrm{W} / \mathrm{m}^{2} \cdot \mathrm{K}\right) \\ \mathrm{k}_{\mathrm{f}} & \text { Thermal conductivity of fluid }(\mathrm{W} / \mathrm{m} \cdot \mathrm{K}) \\ \mathrm{k}_{\mathrm{w}} & \text { Thermal conductivity of wall }(\mathrm{W} / \mathrm{m} \cdot \mathrm{K}) \\ \mathrm{k}_{\mathrm{ex}} & \text { Thermal conductivity of heat exchanger } \\ & \text { (W/m } \cdot \mathrm{K}) \\ \mathrm{L}_{\text {loop }} & \text { Total loop length }(\mathrm{m}) \\ \mathrm{L}_{\mathrm{H}} & \text { Loop height }(\mathrm{m}) \\ \mathrm{N}_{\mathrm{G}} & \text { Length to diameter ratio }(\mathrm{L} \text { loop } / \mathrm{D}) \\ \mathrm{Re}_{\mathrm{d}} & \text { Reynolds number } \\ \dot{Q} & \text { Volumetric heat generation }\left(\mathrm{W} / \mathrm{m}^{3}\right) \\ \mathrm{v}_{\mathrm{f}} & \text { Fluid velocity }(\mathrm{m} / \mathrm{s}) \\ \mathrm{V}_{\mathrm{w}} & \text { Wall volume }\left(\mathrm{m}^{3}\right) \\ \mathrm{V}_{\mathrm{ex}} & \text { Heat exchanger fluid volume }\left(\mathrm{m}^{3}\right) \\ \beta_{\mathrm{av}} & \text { Thermal volumetric expansion } \\ \mu_{\mathrm{f}} & \text { coefficient }\left(\mathrm{K}{ }^{-1}\right) \\ v & \text { Dynamic viscosity of fluid }(\mathrm{kg} / \mathrm{m} \cdot \mathrm{s}) \\ \rho_{\mathrm{f}} & \text { Kinematic viscosity of fluid }\left(\mathrm{m}{ }^{2} / \mathrm{s}\right) \\ \rho_{\mathrm{w}} & \text { Fluid density }\left(\mathrm{kg} / \mathrm{m}^{3}\right) \\ \rho_{\mathrm{ex}} & \text { Density of wall }\left(\mathrm{kg} / \mathrm{m}^{3}\right) \\ & \text { Coolant density }\left(\mathrm{kg} / \mathrm{m}^{3}\right) \\ & \end{array}$

\section{References}

[1] R K Sinha and A Kakodkar, Nucl. Eng. Des. 236, 683 (2006)

[2] E H Ridouane, C M Danforth and D L Hitt, Int. J. Heat. Mass. Tran. 53, 76 (2010)

[3] M Maiani, W J M d Kruijf and W Ambrosini, Int. J. Heat. Fluid. Fl. 24, 853 (2003)

[4] H Nishikawa, K Matsumura, S Okino, T Watanabe and F Suda, J. Therm. Sci. Techn. 10, 15 (2015)

[5] L Chen, X R Zhang, H Yamaguchi and Z S (Simon) Liu, Int. J. Heat. Mass. Tran. 53, 4101 (2010)
[6] P K Vijayan, M Sharma, D S Pilkhwal, D Saha, R K Sinha, V K Bhojwani and N K Sane, Experimental and Numerical Investigations on the Nature of the Unstable Oscillatory Flow In A Single-Phase Natural Circulation Loop, in XVII National and VI ISHMT/ASME heat and mass transfer conference (IGCAR, Kalpakkam, 2004)

[7] P K Vijayan, M H Bade, D Saha, R K Sinha and V V Raj, A Generalized Flow Correlation For SinglePhase Natural Circulation Loops, in XVII National and VI ISHMT/ASME heat and mass transfer conference (IGCAR, Kalpakkam 2004)

[8] A K Nayak, P K Vijayan, D Saha and V V Raj, Math. Comput. Model. 22, 77 (1995)

[9] G Cammarata, A Fichera and A Pagano, Int. Comm. Heat. Mass. Tran. 27, 1077 (2000)

[10] P K Vijayan, H Austregesilo and V Teschendorff, Nucl. Eng. Des. 155, 623 (1995)

[11] M Sharma, D S Pilkhwal, P K Vijayan, D Saha and R K Sinha, Nucl. Eng. Des. 240, 588 (2010)

[12] A K Nayak, M R Gartia and P K Vijayan, Nucl. Eng. Des. 239, 526 (2009)

[13] S K Mousavian, M Misale, F D'Auria and M A Salehi, Ann. Nucl. Energy 31, 1177 (2004)

[14] G Desrayaud, A Fichera and G Lauriat, Appl. Therm. Eng. 50, 187 (2013)

[15] G Desrayaud, A Fichera and M Marcoux, Int. J. Heat. Fluid. Fl. 27, 154 (2006)

[16] W Zhang, S Tan, P Gao, Z Wang, L Zhang and H Zhang, Ann. Nucl. Energy 65, 1 (2014)

[17] R Saha, Experimental and Numerical Study of a SinglePhase Square Natural Circulation Loop (Jadavpur University, Kolkata, 2013)

[18] R Saha, S Sen, S Mookherjee, K Ghosh, A Mukhopadhyay and D Sanyal, J. Heat. Trans. 137, 121010 (2015)

[19] N Marwan, J F Donges, Y Zou, R V Donner and J Kurths, Phys. Lett. A 373, 4246 (2009)

[20] R Rusinek and K Zaleski, Meccanica 51, 1275 (2016)

[21] N Marwan, Eur. Phys. J. Special. Topics 164, 3 (2008)

[22] L Kabiraj, A Saurabh, H Nawroth and C O Paschereit, AIAA. J 53, 1199 (2015)

[23] N Marwan, M C Romano, M Thiel and J Kurths, Phys. Rep. 438, 237 (2007)

[24] J P Eckmann, S O Kamphorst and D Ruelle, Europhys. Lett. 4, 973 (1987)

[25] M Llop, N Gascons and F X Llauró, Int. J. Multiphas. Flow 73, 43 (2015)

[26] S Datta, S Mondal, A Mukhopadhyay, D Sanyal and S Sen, Combust. Theor. Model. 13, 17 (2009) 